\title{
Accuracy of the iPhysioMeter Heart Rate Monitoring Application
}

\section{Suzanne Greenwalt',2, Ellen Bogue ${ }^{3}$, Angelo Bologna4, Mary Alicia Bowden5, Li Yin Goode6, Rachel Wolfe $^{7}$}

\author{
${ }^{1}$ Belmont University, Nashville, TN, USA \\ ${ }^{2}$ A.T. Still University, Mesa, AZ, USA \\ ${ }^{3} \mathrm{CHI}$ Health Rehabilitation Hospital, Tacoma, WA, USA \\ ${ }^{4}$ Integrity Rehab Group, Bartlett, TN, USA \\ ${ }^{5}$ Encompass Health Rehabilitation of North Memphis, Memphis, TN, USA \\ ${ }^{6}$ Cora Physical Therapy, Tennessee Orthopaedic Clinics-Lenoir City Physical Therapy, Lenoir City, TN, USA \\ ${ }^{7}$ BenchMark Physical Therapy, Pulaski, TN, USA \\ Email: suzanne.greenwalt@belmont.edu, sgreenwalt@atsu.edu, eebogue@hotmail.com, angelojbologna@gmail.com, \\ mary.bowden@encompasshealth.com, lgoode@corahealth.com, goodely@tocdocs.com,rwolfe@benchmarkpt.com
}

How to cite this paper: Greenwalt, S., Bogue, E., Bologna, A., Bowden, M.A., Goode, L.Y. and Wolfe, R. (2019) Accuracy of the iPhysioMeter Heart Rate Monitoring Application. Open Access Library Journal, 6: e5210.

https://doi.org/10.4236/oalib.1105210

Received: January 28, 2019

Accepted: February 25, 2019

Published: February 28, 2019

Copyright () 2019 by author(s) and Open Access Library Inc.

This work is licensed under the Creative Commons Attribution International License (CC BY 4.0).

http://creativecommons.org/licenses/by/4.0/

\section{(c) (i) Open Access}

\begin{abstract}
Standard heart rate (HR) monitoring pulse oximeters use light sensors to acquire HR data through photoplethysmography (PPG). By employing a smartphone's camera and processing software, a smartphone can measure HR with PPG. The current study investigated the accuracy of the iPhysioMeter smartphone app in measuring heart rate before, during, and immediately after exercise on a treadmill to determine whether exercise and associated increases in heart rate affected the app's accuracy. Twenty-eight participants (aged 21 - 29 years) completed the study. Using a repeated-measures design, HR was measured with the iPhysioMeter app on an iPhone 6s, a 12-lead electrocardiogram (EKG), and a pulse oximeter. The first three stages of the Bruce treadmill protocol were used for standardized exercise testing. Significant differences were found between elevated HR readings from the EKG and pulse oximetry at Bruce 3 and between the iPhysioMeter app and EKG at Bruce 2 and Bruce 3. No significant differences were found for resting values or for Bruce 1 for any of the HR devices. Results suggested HR monitoring apps are an attractive alternative to expensive HR devices because of their usability and affordability. However, because the iPhysioMeter app had limited accuracy with elevated HR during exercise, caution should be used if relying on the app to monitor HR during exercise.
\end{abstract}

\section{Subject Area}

Public Health 


\section{Keywords}

Heart Rate Monitoring, Heart Rate Apps, iPhysioMeter, Heart Rate with Exercise

\section{Introduction}

Because they measure how well the body is performing essential cardiovascular functions, monitoring vital signs is an important part of any health care provider's job. Commonly collected vital signs are body temperature, HR, blood pressure, and respiratory rate [1]. Recent technologic advances allow smartphones to measure vital signs, and studies have investigated the use of smartphones for measuring heart rate, blood pressure, and respiratory rate [2]-[11]. Using smartphones to identify arrhythmias, such as atrial fibrillation and tachycardia, has also been investigated [12] [13] [14]. The most common vital sign assessed with smartphone apps is heart rate. These apps are readily accessible and frequently used; therefore, it is essential to assess their accuracy.

In addition to manual pulse readings, pulse oximeters are also commonly used to monitor heart rate because they are easy to use and cost effective. Conventional pulse oximeters are placed around a finger, toe, or earlobe, and the accuracy of these is comparable across sites and side of the body [15]. Pulse oximeters operate through photoplethysmography (PPG), which is a low-cost technique that measures oxygen perfusion and heart rate with a photodetector. They can function through two different methods: transmissive, where the photoemitter and photodetector are placed separately from each other, and reflective, where the photoemitter and photodetector are at the same site. Despite their benefits and unlike electrocardiograms (EKGs), pulse oximeters cannot show electric cardiac events. As such, they do not offer a full picture of cardiovascular activity [16].

Smartphone apps that measure heart rate use reflective PPG technology, where the camera and flash of the smartphone are used to measure the quantity of light absorbed into peripheral tissues as blood pulses through capillaries [2]. Another benefit of these apps is that they assess heart rate and oxygen perfusion. Multiple studies have tested finger PPG technology in smartphones with mostly accurate results. For example, Losa-Iglesias et al. [4] found that the Heart Rate Plus app had $97 \%$ accuracy in determining heart rate in healthy individuals without diagnosed heart problems when compared with manual radial pulse and pulse oximeter measures. Research on iPhysioMeter, an app that uses technology similar to Heart Rate Plus, has shown that the app can accurately measure heart rate when compared with EKG during mental stress tests and in the presence of controlled horizontal and vertical body movements [5] [6]. However, initial research validating the accuracy of iPhysioMeter was performed by the app's developers. Smartphone PPG technology can also accurately adjust for relatively 
small changes in heart rate and can accommodate normal and low lighting conditions [7] [8]. A variety of smartphone models have been used in this research, such as HTC, iPhone 4, Nokia, and Samsung Galaxy [2]. Taken altogether, research has demonstrated the ability of smartphones to accurately measure heart rate, even after adjusting for confounding factors, such as light and movement [2] [6] [7] [8]. As such, smartphone technology enables greater involvement in personal health monitoring by providing consumers with a valid tool for detecting heart rate.

Despite advancements in smartphone technology, however, heart rate monitoring apps still have limitations. Ho et al. [9] found apps had trouble measuring heart rates above $120 \mathrm{bpm}$ or in children aged 0 - 15 years. Some apps were affected by the body site where heart rate was measured. McManus, Lee and Maitas [14] found apps measuring heart rates above $200 \mathrm{bpm}$ in children were significantly less accurate and reliable than those detecting lower heart rates. Pelegris, Banitsas, Orbach and Marias [8] noted the biggest problems with accuracy were user error, low light (though their average error only changed from $4.13 \%$ to $4.67 \%$ ), and poor hardware (cited Android's 5fps framerate and processing speed). Lázaro, Nam, Gil, Lauguana and Chon [3] suggested smartphone-collected PPG data were more vulnerable to outside interference (e.g., ambient lighting or finger pressure on the camera) than traditional PPG data from pulse oximetry. Cumulatively, these inaccuracies in heart rate monitoring apps highlight systematic errors in measurement that, once identified, can be accounted for in programming or usage [3] [8] [9] [12]. Correctly identifying causes of error can create more accurate measurements for users and ultimately lead to the development of more valid apps.

Smartphone technology may also be useful to detect heart rate irregularities, such as fibrillation and tachycardia [12] [13] [14]. Research on this technology has been mixed; some studies found 100\% accuracy in detecting irregularities but others were unsuccessful in detecting irregularities [12] [13] [14]. For example, Wackel et al. [12] found that apps were not as accurate when dealing with tachycardia that was greater than $200 \mathrm{bpm}$, while McManus et al. [14] found a novel smartphone application that was $96.5 \%$ - 100\% accurate in detecting atrial fibrillation and irregular pulse. It should be noted that this technology is evolving and may not be as accurate as traditional measurement methods.

Although smartphone technology may be useful for monitoring heart rate, to our knowledge, no studies have investigated the accuracy of heart rate smartphone apps at elevated heart rates, such as during or after exercise. Consequently, we considered measurement of heart rate during and after exercise as an area worthy of investigation. If smartphone apps for measuring heart rate are to be used and interpreted by medical and non-medical users, then the apps need to be accurate and their failure points defined. Therefore, the purpose of the current study was to investigate the accuracy of the iPhysioMeter smartphone app in measuring heart rate before, during, and immediately after exercise to deter- 
mine whether exercise and associated increases in heart rate affected the app's accuracy when compared with EKG and pulse oximetry. We included EKG as a gold standard measurement for heart rate data and pulse oximetry as a comparator because of its widespread use in the clinical setting.

\section{Methods}

\subsection{Participants}

The number of participants for the current study was determined after a pilot study of 10 individuals and power analysis of that data was performed. Therefore, 28 participants ( 9 males, 19 females, age range $=21-29$ years) were recruited from in and around a local university through word-of-mouth and social media. Participation was voluntary and non-random convenience sampling was used. Potential participants were excluded if they had been diagnosed with a current and unmanaged heart arrhythmia or other heart condition, pregnancy, diabetes, hypertension, or adverse reactions to exercise as noted on the study's health history questionnaire. They were also excluded if they displayed signs of illness or injury on the day of data collection. Potential participants with a positive diagnosis of a heart condition that had been medically or surgically corrected were allowed to participate in the study provided their condition did not interfere with data collection (e.g., app was unable to detect a pulse) and they were cleared to perform exercise. Participants had to be able to complete $9 \mathrm{mi}$ nutes of the Bruce treadmill protocol at increasing speeds and inclines. All participants were asked to wear athletic attire, including tennis shoes; and males were asked to come with a clean-shaven chest. Approval for the study was obtained from the local institutional review board before the recruitment process. All participants were provided with a description of the exercise protocol and the process for measuring heart rates, and they signed an informed consent form before data collection.

\subsection{Instrumentation}

The following instrumentation was used in the current study: Apple iPhone 6S (Apple, Inc., Cupertino, CA), iPhysioMeter smartphone application (Matsumura and Yamakoshi, 2012), 12-lead EKG VMAX Carefusion metabolic cart (Vyaire Medical, Illinois), pulse oximeter (Nonin Medical Inc., Plymouth, Minnesota), and a treadmill programmed with the Bruce treadmill protocol. A standard sphygmomanometer and stethoscope were used before and after testing to monitor participant blood pressure recovery. Only the first three stages of the Bruce treadmill protocol were used in the current study as a standardized exercise protocol to increase participant heart rate; this protocol is designed to increase heart rate without requiring participants to run. The first stage (Bruce 1) required participants to walk for 3 minutes at a speed of $1.7 \mathrm{mph}$ with a $10 \%$ incline, the second stage (Bruce 2) required participants to walk for 3 minutes at a speed of $2.5 \mathrm{mph}$ with a $12 \%$ incline, and the third stage (Bruce 3 ) required par- 
ticipants to walk for 3 minutes at a speed of $3.4 \mathrm{mph}$ with a $14 \%$ incline.

\subsection{Design and Data Collection}

When participants arrived for data collection, the testing procedure was explained to them. Participants then completed a health history questionnaire to ensure they could participate. The questionnaire asked for basic demographic information (sex, age, height, weight) and about medical conditions or heart medications, exercise restrictions, and current illness. Participants were then connected to a 12-lead EKG in a separate, private room by two study investigators. The same investigator placed the leads on every participant. Baseline blood pressure measurements were taken to monitor for safety and recovery before and after testing. Participants were instructed to hold the iPhone in their right hand with their index finger covering the phone's camera lens per iPhysioMeter app instructions. The phone was secured in place with a Velcro strap around the back of the participant's hand. The pulse oximeter was placed on the participant's left index finger.

Initial heart rate measurements were taken at the start of data collection with the participant in supine position (Initial 1) using the iPhysioMeter app, EKG, and pulse oximeter concurrently. Participants were then moved to a sitting position (Initial 2), and all measurements were repeated. Participants were moved to a standing position (Initial 3), and all measurements were again repeated. After initial measurements, participants moved to the treadmill and began the Bruce protocol. During the last minute of each of the three stages, heart rate measurements were collected concurrently from all three devices. After completing the three stages of the Bruce protocol, participants had a 3-minute cool down period of walking at a speed of $1.5 \mathrm{mph}$ with a $0 \%$ incline. The treadmill was then stopped, and heart rate measurements (Post) were again taken concurrently from all three devices. Before leaving the lab and ending their data collection session, participants had final heart rate measurements and blood pressure readings taken to ensure proper recovery. All participants remained in the lab until baseline levels were reached.

Patient safety was monitored during the testing sessions. The Borg Scale of Perceived Exertion was administered at the beginning of each phase. Symptoms were also monitored and testing was stopped if participants experienced any adverse responses at any point, such as angina, light-headedness, extreme shortness of breath, or other adverse symptoms. Testing was also stopped when requested by the participant or when the participant reached the age-predicted maximum heart rate. The blood pressure readings obtained before and after testing were used for monitoring participant safety but were not used in the data analysis.

\subsection{Data Analysis}

Heart rate data collected during the study was analyzed using SPSS version 21. 
Descriptive statistics of heart rate, including means, standard deviations and ranges, are reported. A repeated-measures ANOVA was used to test for differences between stages $(1-7)$ and an interaction between device (EKG, Pulse Ox, and App) and stage. A random participant effect was included to account for common effects from an individual. Mauchly's criterion was used to test whether Huynh-Feldt conditions on the covariance are too restrictive and Greenhouse-Geisser was used as necessary to adjust $p$-values. Post-hoc tests included separate repeated-measures ANOVA for each stage to confirm the location of the significant differences in the interaction between stage and device. Further post-hoc tests using paired t-tests were used to confirm the location of the significant differences among devices within stages. Bonferroni correction was used to adjust the significance level of the paired $\mathrm{t}$-tests to alpha $=0.008$. For all other tests, statistical significance was defined as $P<0.05$.

\section{Results}

Heart rate data for all time points are summarized in Table 1. Mauchley's criterion indicated Huynh-Feldt conditions are too restrictive $(P<0.001)$, thus Greenhouse-Geisser adjusted $p$-values are reported. Heart rate increased from Initial 1 (iPhysioMeter, $74 \mathrm{bpm}$; EKG, $70 \mathrm{bpm}$; pulse oximeter, $71 \mathrm{bpm}$ ) to Bruce 3 (iPhysioMeter, 127 bpm; EKG, 165 bpm; pulse oximeter, $144 \mathrm{bpm})(P<0.05)$. A significant interaction between the device and protocol stage was confirmed $(P<0.001)$.

Differences between devices were found for Bruce $2(P=0.004)$ and Bruce 3 $(P<0.001)$ as shown in Table 1. Significant differences were found between $\mathrm{EKG}$ and the App in Bruce $2($ difference $=15 \mathrm{bpm})$ and $3($ difference $=38 \mathrm{bpm})$ and between EKG and Pulse Oximetry in Bruce 3 (difference $=21 \mathrm{bpm}$ ). No significant differences were noted between devices for measurements taken at rest, during stage one of the Bruce protocol, or following exercise.

\section{Discussion}

The EKG is considered the gold standard for measuring heart rate, but it can be expensive to use and not readily accessible to the public. Therefore, alternative measuring methods need to be explored. Smartphone apps are an easy, inexpensive way to measure heart rate, but research is limited about their accuracy of heart rate measurements during periods of elevated heart rate. In the current study, we compared the accuracy of iPhysioMeter, a smartphone app, during exercise with EKG and pulse oximetry. While all three devices appeared to be consistent when study participants were at rest, significant differences were found between the devices during the second and third stages of the Bruce treadmill protocol. Specifically, iPhysioMeter became increasingly inaccurate as participant heart rate increased. As such, heart rates above $134 \mathrm{bpm}$ (measured by EKG at Bruce 2) may not be accurately measured by iPhysioMeter during exercise. Our results also suggested that pulse oximetry may become inaccurate at higher heart rates. Given these results, clinicians should use the iPhysioMeteror 
Table 1. Heart rate data of study participants $(n=28)$ measured by the iPhysioMeter iPhone app, electrocardiogram (EKG), and pulse oximetry at all study time points.

\begin{tabular}{cccccccc}
\hline Stage & \multicolumn{2}{c}{ iPhysioMeter app } & \multicolumn{2}{c}{ EKG } & \multicolumn{2}{c}{ Pulse oximeter } & $P$ \\
\hline & $M(\mathrm{SD})$ & Range & $M(\mathrm{SD})$ & Range & $M(\mathrm{SD})$ & Range & \\
\hline Initial 1 & $74(11)$ & $48-98$ & $70(11)$ & $46-94$ & $71(12)$ & $47-94$ & $P<0.05$ \\
Initial 2 & $79(12)$ & $55-112$ & $78(13)$ & $48-111$ & $76(12)$ & $48-109$ & $P<0.05$ \\
Initial 3 & $87(13)$ & $62-124$ & $83(13)$ & $60-121$ & $84(14)$ & $54-24$ & $P<0.05$ \\
Bruce 1 & $103(11)$ & $82-133$ & $108(13)$ & $78-134$ & $106(12)$ & $72-133$ & $P<0.05$ \\
Bruce 2 & $119(17)$ & $98-156$ & $134(16)$ & $98-171$ & $129(16)$ & $103-166$ & $P=0.004^{*}$ \\
Bruce 3 & $127(23)$ & $86-163$ & $165(18)$ & $102-187$ & $144(25)$ & $102-184$ & $P<0.001^{*}$ \\
Post & $115(18)$ & $68-150$ & $118(20)$ & $63-155$ & $116(19)$ & $61-154$ & $P<0.05$ \\
\hline
\end{tabular}

Note: Bruce 1 = heart rate measured in last minute of first stage of Bruce treadmill protocol; Bruce 2 = heart rate measured in last minute of second stage of Bruce treadmill protocol; Bruce $3=$ heart rate measured in last minute of third stage of Bruce treadmill protocol; Initial 1 = heart rate measured in supine; Initial $2=$ heart rate measured in sitting; Initial $3=$ heart rate measured in standing; Post $=$ heart measured after cool down. $P$ by post-hoc repeated-measures ANOVA. ${ }^{*}=$ significant difference between devices.

pulse oximetry with caution when monitoring the heart rates of patients during exercise because these methods may provide inaccurate results at higher heart rates.

The current study had several limitations. We used a controlled exercise protocol and secured the smartphone to the participant's hand, which may not accurately reflect how iPhysioMeter would be used by the general population. Other factors may have also impacted results, such as a cold testing environment, calibration issues, dynamic movement when exercising, and EKG lead placement (including dislodgement during testing). Occasionally, iPhysioMeter and the pulse oximeter had difficulty acquiring a reading, which resulted in missing data and may have caused inconsistent heart rate readings. However, no outlier data were removed from the analysis. The decision to keep all data points was meant to reflect how the app would be used by the general population. In some instances, apps have technical difficulties; therefore, the decision was made to ensure realistic results.

\section{Conclusion}

A free heart rate monitoring smartphone app is an attractive alternative for individuals who do not have access to other heart rate monitoring devices. However, results of the current study suggest that the iPhysioMeter app has limitations at the elevated heart rates seen during exercise. Therefore, the accuracy of the application does not compare well with the gold standard EKG. Additional studies should investigate other vital sign smartphone apps and devices. Further, as technology continues to advance, additional research is needed to establish more accurate heart rate monitoring hardware and software.

\section{Acknowledgements}

We are grateful to Deb Goggin, Research Support and Scientific Writer at A.T. 
Still University and Vanessa K. Pazdernik, Biostatistician and Research Support at A.T. Still University for their support throughout this work.

\section{Conflicts of Interest}

The authors have no conflicts of interest to report. The current study received no grants from funding agencies in the public, commercial or not-for-profit sectors. There was no financial support for this study that could have influenced its outcomes.

The authors alone are responsible for the writing and content, and confirm that this manuscript has been read and approved by all named authors, and that there are no other persons who satisfied the criteria for authorship but are not listed. We understand that the Corresponding Author is the sole contact for the Editorial.

\section{References}

[1] Storm-Versloot, M.N., Verweij, L., Lucas, C., et al. (2014) Clinical Relevance of Routinely Measured Vital Signs in Hospitalized Patients: A Systematic Review. Journal of Nursing Scholarship, 46, 39-49. https://doi.org/10.1111/jnu.12048

[2] Kurylyak, Y., Lamonaca, F. and Grimaldi, D. (2012) Smartphone-Based Photoplethysmogram Measurement. 135-164.

http://books.google.com/books?hl=en\&lr=\&id=F7yzfrJtwKgC\&oi=fnd\&pg=PA135 $\& \mathrm{dq}=$ Smartphone-Based+Photoplethysmogram+Measurement\&ots=woysNsIIMA \&sig=BjtNoqdM_NcKEqVISFLP1yeth2I

[3] Lázaro, J., Nam, Y., Gil, E., Laguna, P. and Chon, K.H. (2015) Respiratory Rate Derived from Smartphone-Camera-Acquired Pulse Photoplethysmographic Signals. Physiological Measurement, 36, 2317-2333. https://doi.org/10.1088/0967-3334/36/11/2317

[4] Losa-Iglesias, M.E., Becerro-de-Bengoa-Vallejo, R. and Becerro-de-Bengoa-Losa, K.R. (2014) Reliability and Concurrent Validity of a Peripheral Pulse Oximeter and Health-App System for the Quantification of Heart Rate in Healthy Adults. Health Informatics Journal, 22, 1-9. https://doi.org/10.1177/1460458214540909

[5] Matsumura, K. and Yamakoshi, T. (2013) iPhysioMeter: A New Approach for Measuring Heart Rate and Normalized Pulse Volume Using Only a Smartphone. Behavior Research Methods, 45, 1272-1278.

https://doi.org/10.3758/s13428-012-0312-Z

[6] Matsumura, K., Rolfe, P., Lee, J. and Yamakoshi, T. (2014) iPhone 4s Photoplethysmography: Which Light Color Yields the Most Accurate Heart Rate and Normalized Pulse Volume Using the iPhysioMeter Application in the Presence of Motion Artifact? PLOS ONE, 9, e0091205.

https://doi.org/10.1371/journal.pone.0091205

[7] Lakens, D. (2013) Using a Smartphone to Measure Heart Rate Changes during Relived Happiness and Anger. IEEE Transactions on Affective Computing, 4, 238-241. https://doi.org/10.1109/T-AFFC.2013.3

[8] Pelegris, P., Banitsas, K., Orbach, T. and Marias, K. (2010) A Novel Method to Detect Heart Beat Rate Using a Mobile Phone. 2010 Annual International Conference of the IEEE Engineering in Medicine and Biology Society, Buenos Aires, 31 August-4 September 2010, 5488-5491. https://doi.org/10.1109/IEMBS.2010.5626580 
[9] Ho, C.L., Fu, Y.C., Lin, M.C., Chan, S.C., Hwang, B. and Jan, S.L. (2014) Smartphone Applications (apps) for Heart Rate Measurement in Children: Comparison with Electrocardiography Monitor. Pediatric Cardiology, 35, 726-731. https://doi.org/10.1007/s00246-013-0844-8

[10] Scully, C.G., Lee, J., Meyer, J., et al. (2012) Physiological Parameter Monitoring from Optical Recordings with a Mobile Phone. IEEE Transactions on Biomedical Engineering, 59, 303-306. https://doi.org/10.1109/TBME.2011.2163157

[11] Chandrasekaran, V., Dantu, R., Jonnada, S., Thiyagaraja, S. and Subbu, K.P. (2013) Cuffless Differential Blood Pressure Estimation Using Smart Phones. IEEE Transactions on Biomedical Engineering, 60, 1080-1089.

[12] Wackel, P., Beerman, L., West, L. and Arora, G. (2014) Tachycardia Detection Using Smartphone Applications in Pediatric Patients. The Journal of Pediatrics, 164, 1133-1135. https://doi.org/10.1016/j.jpeds.2014.01.047

[13] Lee, J., Reyes, B.A., McManus, D.D., Mathias, O. and Chon, K.H. (2012) Atrial Fibrillation Detection Using a Smart Phone. Annual International Conference of the IEEE Engineering in Medicine and Biology Society, 2012, 1177-1180. https://doi.org/10.1109/EMBC.2012.6346146

[14] McManus, D.D., Lee, J., Maitas, O., et al. (2013) A Novel Application for the Detection of an Irregular Pulse Using an iPhone $4 \mathrm{~S}$ in Patients with Atrial Fibrillation. Hear Rhythm, 10, 315-319. https://doi.org/10.1016/j.hrthm.2012.12.001

[15] Allen, J. and Murray, A. (2000) Similarity in Bilateral Photoplethysmographic Peripheral Pulse Wave Characteristics at the Ears, Thumbs and Toes. Physiological Measurement, 21, 369-377. https://doi.org/10.1088/0967-3334/21/3/303

[16] Reisner, A., Shaltis, P.A., McCombie, D. and Asada, H.H. (2008) Utility of the Photoplethysmogram in Circulatory Monitoring. Anesthesiology, 108, 950-958. https://doi.org/10.1097/ALN.0b013e31816c89e1 